\title{
Outflows in nearby AGNs
}

\author{
Thaisa Storchi-Bergmann \\ Instituto de Física - UFRGS, Campus do Vale, CP 15051, Porto Alegre, RS, Brasil \\ email: thaisa@ufrgs.br
}

\begin{abstract}
I report results of kinematic studies of the Narrow-Line Region (NLR) of nearby Active Galactic Nuclei (AGN) from integral field spectroscopy (IFS) obtained with the Gemini Telescopes, including mass outflow rates and corresponding kinetic power. The IFS has allowed the construction of velocity channel maps which provide a better coverage of the gas kinematics and do not support the presence of acceleration up to hundred parsec scales in the NLR as found in previous studies based solely on centroid velocity maps.
\end{abstract}

Keywords. galaxies: active, galaxies: jets, galaxies: nuclei, galaxies: kinematics and dynamics, galaxies: ISM, infrared: galaxies

Outflows are ubiquitous among AGN (Veilleux et al. 2005) and provide the feedback which regulates the M- $\sigma$ relation (Di Matteo et al. 2005). The quantification of the mass and energy deposited by these outflows is thus a fundamental ingredient for the understanding of galaxy evolution. With the goal of mapping and quantifying the feedback from nuclear outflows we have been observing the nuclear region of nearby AGN using Integral Field Units (IFU) at the Gemini telescopes. In the optical, we have used the Gemini Multi-Object Spectrograph (GMOS) IFU, while in the near-infrared (near-IR), we have used the Near-Infrared Integral Field Spectrograph (NIFS) with the adaptive optics module ALTAIR. In the optical, the angular resolution is limited by the atmospheric seeing (typically 0.6 arcsec), while in the near-IR, the adaptive optics provides a much better angular resolution $(\approx 0.1 \mathrm{arcsec})$.

The main results of our studies are:

- Ionized gas emission is not restricted to the outflowing region, indicating that the obscuring dusty structure Riffel et al. (2009) - the torus of the Unified Model (Antonucci \& Miller 1985) - is clumpy (Elitzur 2006), allowing the escape of radiation along its equatorial plane. This radiation then ionizes gas from the galaxy plane.

- While the stellar kinematics is dominated by rotation in the galaxy plane (Barbosa et al. 2006), the gas kinematics shows both rotation and outflow. Rotation is observed mainly in the ionized gas from the galaxy plane, while the outflow is observed in an elongated or biconical structure usually following the radio structure.

- Different phases of the gas show different kinematics: while in the ionized gas the outflowing component is usually dominating, in the molecular gas $\left(\mathrm{H}_{2}\right)$ the dominant component is rotation, revealing that the $\mathrm{H}_{2}$ gas is more settled in the galaxy plane, being also observed in inflow (Riffel et al. 2008).

- In many cases there is a close association between the radio structure and the gas kinematics, such as flux enhancements at the location of radio knots and increased velocity dispersion surrounding these knots (Barbosa et al. 2009; Riffel et al. 2006).

- Centroid velocity maps usually show highest velocities (typically $\sim 200-300 \mathrm{kms}$ ) away from the nucleus, suggesting acceleration along the NLR up to $\sim 100 \mathrm{pc}$ from the nucleus. Our data suggest that this result may be due to projection effects which combine emission from the outflow with that from the galaxy plane. As a result, the centroid velocities show a shift of the brightest component from that in the galactic disk at zero 


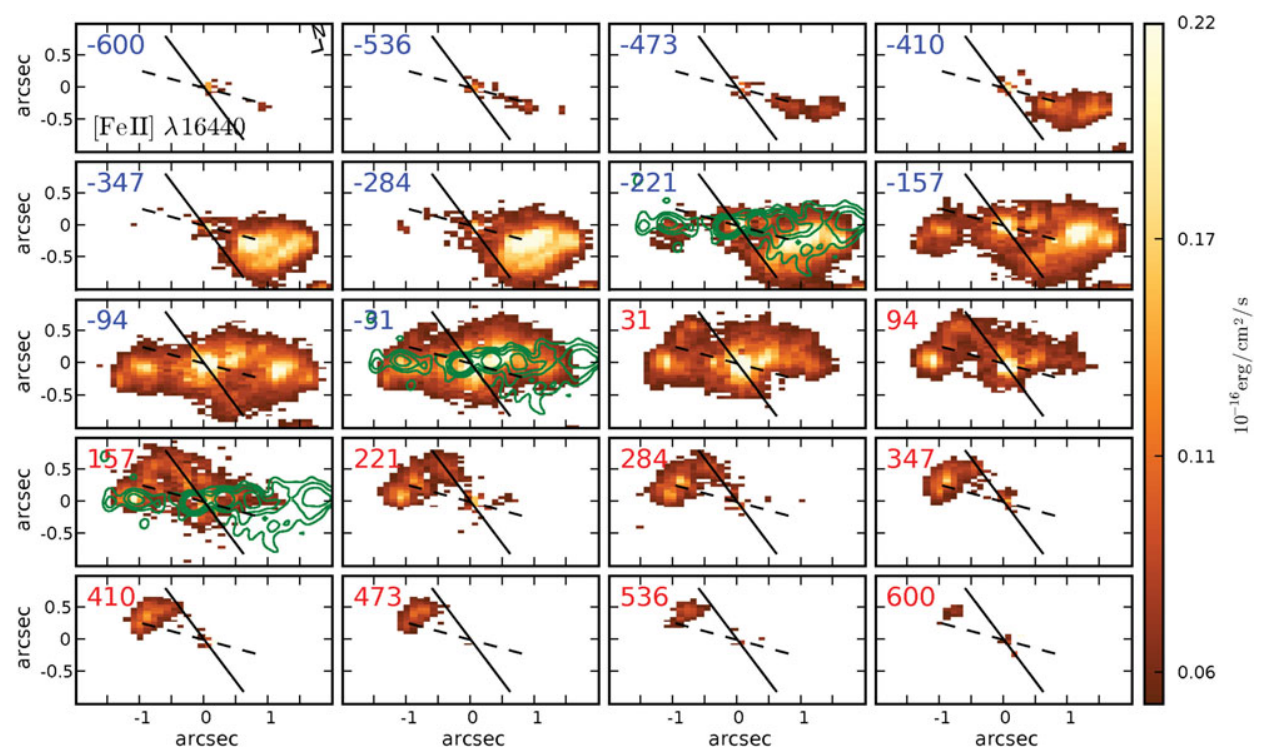

Figure 1. Channel maps obtained by integrating the flux within velocity bins of $63 \mathrm{kms}^{-1}$ along the [Fe II] $\lambda 1.64 \mu \mathrm{m}$ emission-line profile from the NLR of NGC 4151 (Storchi-Bergmann et al. 2010). The numbers in the upper left corner of each panel are the central velocity of the bin, in $\mathrm{kms}^{-1}$ relative to systemic. The continuous line shows the orientation of the galaxy major axis and the dashed line shows the orientation of the bi-cone which characterize the NLR emission-line flux distributions in this galaxy. Contours are from a radio MERLIN image.

velocity - which dominates near the nucleus, to the higher velocity outflowing component - which dominates outwards.

- Channel maps, on the other hand, usually show the highest velocities close to the nucleus, as illustrated in Fig.1 and do not support acceleration along the NLR. Our data suggest that the NLR clouds are accelerated very close to the nucleus (within $\approx 10 \mathrm{pc}$ ), and then the flow moves at essentially constant velocity (Storchi-Bergmann et al. 2010).

- Mass outflow rates range from $10^{-2}$ to $1 \mathrm{M}_{\odot}$ corresponding to $\sim 10-200$ times the accretion rate to the AGN, implying that the origin of the outflow is gas from the galaxy plane being pushed by a radio jet or accretion disk wind. The kinetic power of the outflow is usually $\leqslant 10^{-3}$ times the bolometric luminosity, which is small compared with the value of $\sim 5$ derived for LINERs, as presented by Nemmen et al. (2010) at this Conference.

\section{References}

Antonucci, R. R. \& Miller, J. S. 1985, ApJ 297, 621

Barbosa, F. K. B. et al. 2006, MNRAS 371, 170

Barbosa, F. K. B. et al. 2009, MNRAS, 396, 2

Di Matteo, T., Springel, V., \& Hernquist, L. 2005, Nature 433, 604

Elitzur, M. \& Shlosman, I. 2006, ApJ, 648, L101

Nemmen, R., Storchi-Bergmann T., \& Eracleous, M. 2010, in preparation

Riffel R. A., Storchi-Bergmann T., Winge C., \& Barbosa, F. K. B. 2006, MNRAS 373, 2

Riffel, R. A. et al. 2008, MNRAS 385, 1129

Riffel R. A., Storchi-Bergmann T., \& McGregor, P. J. 2009, ApJ 698, 1767

Storchi-Bergmann, T. et al. 2010, MNRAS, in press

Veilleux S., Cecil G., \& Bland-Hawthorn J. 2005, ARA\&SA 43, 769 